\title{
Investigating Context Parameters in Technology Term Recognition
}

\author{
Behrang Q. Zadeh and Siegfried Handschuh* ${ }^{* \dagger}$ \\ *Insight Centre of Data Analytics \\ National University of Ireland, Galway \\ ${ }^{\dagger}$ Department of Computer Science and Mathematics \\ University of Passau, Germany \\ \{behrang.qasemizadeh, siegfried.handschuh\}einsight-centre.org
}

\begin{abstract}
We propose and evaluate the task of technology term recognition: a method to extract technology terms at a synchronic level from a corpus of scientific publications. The proposed method is built on the principles of terminology extraction and distributional semantics. It is realized as a regression task in a vector space model. In this method, candidate terms are first extracted from text. Subsequently, using the random indexing technique, the extracted candidate terms are represented as vectors in a Euclidean vector space of reduced dimensionality. These vectors are derived from the frequency of co-occurrences of candidate terms and words in windows of text surrounding candidate terms in the input corpus (context window). The constructed vector space and a set of manually tagged technology terms (reference vectors) in a $k$-nearest neighbours regression framework is then used to identify terms that signify technology concepts. We examine a number of factors that play roles in the performance of the proposed method, i.e. the configuration of context windows, neighborhood size $(k)$ selection, and reference vector size.
\end{abstract}

\section{Introduction}

Technology terms and their corresponding concepts are part and parcel of any system that tries to capture competitive technological intelligence (QasemiZadeh, 2010; Newman et al., 2014). We propose a method of technology term recognition (TTR) at a synchronic level, i.e., the identification of terms that correspond to technological concepts from a corpus of scientific publications. TTR can be viewed as a kind of automatic term recognition (ATR) task. The input of ATR is a large collection of documents, i.e. a domain-specific corpus, and the output is a terminological resource. The generated terminological resource embraces terms that signify a wide spectrum of concepts in domain knowledge represented by the input corpus. The extracted terms and their corresponding concepts, however, can be further organized in several categories; each category characterizes a group of 'similar' concepts (e.g. technology) in domain knowledge. ${ }^{1}$ TTR, therefore, goes beyond ATR and targets a subset of terms that characterizes the category of technological concepts in domain knowledge (Figure 1).

Establishing a precise definition of technology—and subsequently finding its corresponding terms-is a fundamental problem studied in philosophy of science. The most simplistic definition of technology, perhaps, can be found in a dictionary. For example, Oxford dictionary defines technology as the 'application of scientific knowledge for practical purposes'. As to our understanding, technology terms signal concepts that involve processes-a series of actions taken in order to achieve a particular goal—e.g. as manifested in practical applications of a research. Consequently, technology terms should not be confused with other categories of terms, e.g. terms that signify research subjects or problems. ${ }^{2}$ For example, in computational linguistics literature, both 'language resource' and 'natural language processing' are

\footnotetext{
This work is licensed under a Creative Commons Attribution 4.0 International Licence. Page numbers and proceedings footer are added by the organizers. Licence details: http://creativecommons.org/licenses/by/4.0/

${ }^{1}$ Or, contrariwise, a group of similar concepts can form a category.

${ }^{2}$ Even though, these category of terms are strongly correlated. For instance, a technology may provide a solution for a research problem and can be defined in the scope of a research subject. Therefore, it is important to note that a research problem or a research subject is not a technology.
} 


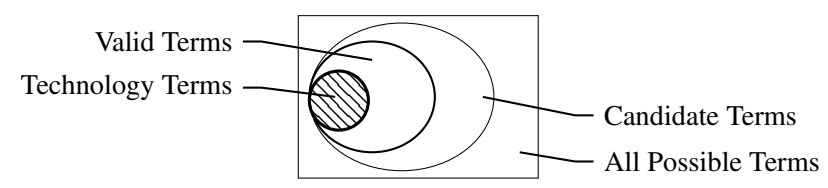

Figure 1: A Venn diagram that illustrates relationships between candidate terms, valid terms and technology terms. ATR targets the identification of valid terms amongst candidate terms. TTR, however, targets the identification of technology terms amongst candidate terms, i.e. a subset of valid terms.

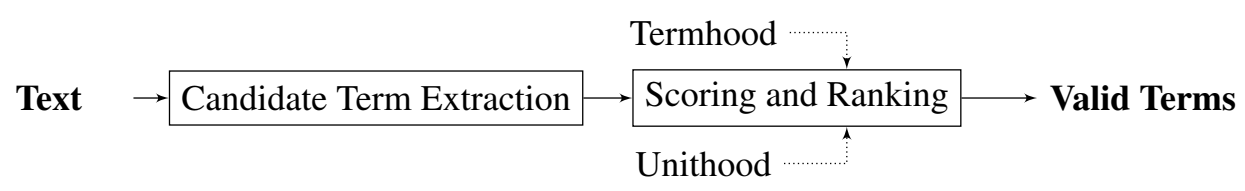

Figure 2: Prevalent architecture of the terminology mining methods.

valid terms; however, we only recognize the latter as a valid technology term. ${ }^{3}$ In this example, 'language resource' signals artefacts such as lexicons and corpora. Although the process of creating these artefacts involves several technologies, we do not consider them-and subsequently the term 'language resource'-as technology.

In the absence of an analytical answer to the question 'what is technology?', we suggest exploiting the context of terms in order to identify technology terms among them. We believe that technology terms tend to appear in similar linguistic contexts. By extending Harris's (1954) distributional hypothesis, we claim that the context of (previously) known technology terms can be modelled and used in order to identify new unknown technology terms. We thus take a distributional approach to the problem of technology term recognition. Consequently, we tie the context of terms to their meaning by quantification of their distributional similarities. We employ vector spaces to model such distributional similarities (Turney and Pantel, 2010). Consequently, the proposed method for TTR is realized as a term classification task in a vector space model (VSM).

The proposed method employs the prevalent mechanism of terminology extraction in the form of a two-step procedure: candidate term extraction followed by term scoring and ranking (Figure 2). Candidate term extraction deals with the term formation and the extraction of term candidates. We employ a linguistic filtering based on part-of-speech $(\mathrm{PoS})$ tag sequences for the extraction of candidate terms. Subsequent to candidate term extraction, a scoring procedure-which can be seen as a semantic weighting mechanism-is employed to indicate how likely it is that a candidate term is a technology term. As suggested in Figure 2, the scoring procedure in ATR usually combines scores that are known as termhood and unithood. Unithood indicates the degree to which a sequence of tokens can be combined to form a complex term (a lexical unit that is made of more than one token). Unithood is, thus, a measure of the syntagmatic relation between the constituents of complex terms: a lexical association measure to identify collocations. ${ }^{4}$ Termhood, on the other hand, 'is the degree to which a stable lexical unit is related to some domain-specific concepts' (Kageura and Umino, 1996). It characterizes a paradigmatic relation between lexical units-either simple (made of one token) or complex terms-and the communicative context that verbalizes domain-concepts. In this paper, in order to simplify the evaluation framework, we assume that the PoS-based approach to candidate term extraction implicitly characterizes the unithood score. The focus is thus on the termhood measure.

We devise a termhood measure to distinguish technology terms from the set of extracted candidate terms. We assume that the association of a term to a technology concept (i.e. what termhood determines) is a kind of paradigmatic relation that can be characterized using the syntagmatic relations of the term and its co-occurred, surrounding words in a context window (Figure 3). Words appeared in context win-

\footnotetext{
${ }^{3}$ Please note that 'natural language processing', in its alternative sense, can also signal a research subject as well as a research problem.

${ }^{4}$ See Evert (2004) on the application of lexical association measures for the identification of collocations.
} 


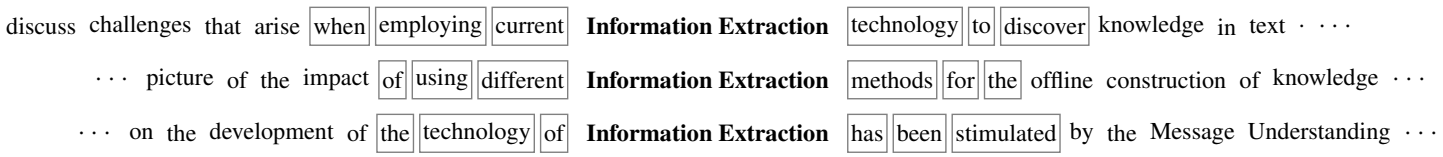

Figure 3: Example of a context window of size 3 that extends around terms: words that are placed in rectangles. In this example, this context-window is shown for the occurrences of the term 'information extraction' in three different sentences.

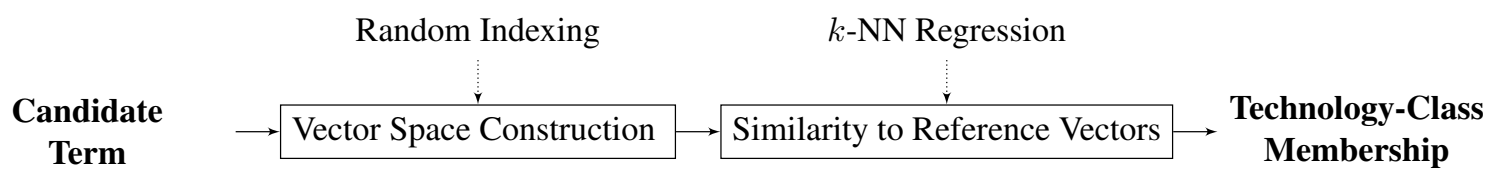

Figure 4: Method for measuring the association of a candidate term to the category of technology terms.

dows are represented by the elements of the standard basis of a vector space. ${ }^{5}$ The frequency of words in context windows of a candidate term (in the whole corpus) then determines the coordinates of the vector that represent the candidate term. To avoid the curse of dimensionality, the VSMs are constructed at reduced dimensionality using the random indexing technique. In this VSM, we characterize the category of technology terms using a set of reference terms, i.e. previously known technology terms. Consequently, the proximity of vectors that represent candidate terms to the vectors that represent reference terms determines the association of candidate terms to the category of technology terms. This association is measured using a $k$-nearest neighbours $(k$-nn) regression algorithm. Figure 4 illustrates the method.

In the proposed technique, finding context window's properties that best characterize technology terms is the major research concern that should be investigated. These properties are the size of the co-occurrence region, the position of a term in the context window and the direction in which the neighbourhood is extended (see Lenci, 2008). To find the most discriminative context window, we construct several VSMs; each VSM represents a context window of a certain configuration (i.e. size, direction and the word order information). We then examine the discriminative power of context windows by reporting the performance of the $k$-nn regression algorithm in these VSMs. Furthermore, to examine the role of the number of reference vectors in the performance of the classification task, we repeat these experiments using various numbers of reference vectors. We report the results of similar evaluation methodology, however, using a $k$-nn voting algorithm in Zadeh and Handschuh (2014b).

In the rest of this paper, we first detail the evaluation framework in Section 2: the employed corpus for the evaluation in Section 2.1, the construction of vector spaces in Section 2.2, the scoring procedure in Section 2.3 and the evaluation methodology in Section 2.4. Subsequently, we report the observed results in Section 3 and conclude in Section 4.

\section{Setting the Scene}

\subsection{Evaluation Corpus}

In order to evaluate the proposed method, we employ the ACL anthology reference corpus (ACL ARC) (Bird et al., 2008) and the ACL reference dataset for terminology extraction and classification (AC RD-TEC) (Zadeh and Handschuh, 2014a). ${ }^{6}$ The ACL ARC has been developed with the aim of providing a platform for benchmarking methods of scholarly document processing. It consists of 10,922 articles that were published between 1965 to 2006 in the domain of computational linguistics. These articles are digitized and enriched with bibliography metadata. The provided resources in the ACL ARC consist of three layers: (a) source publications in portable document format (PDF), (b) automatically extracted text from the articles and (c) bibliographic metadata and citation network. Each of the articles in the collection is assigned to a unique identifier that indicates the source (e.g. journal or conference)

\footnotetext{
${ }^{5}$ That is, informally, each dimension of the vector space.

${ }^{6}$ The ACL RD-TEC can be obtained from the European Language Resources Association, catalogue ELRA-T0375.
} 


\begin{tabular}{|l|l|l|l|l|l|}
\hline Type & Token & Sentence & Paragraph & Section & Publication \\
\hline 704,085 & $36,729,513$ & $1,564,430$ & 510,366 & 92,935 & 10,922 \\
\hline
\end{tabular}

Table 1: Summary statistics of the dataset derived from automatic processing of the ACL ARC.

\begin{tabular}{|c|r|r|r|r|r|r|}
\cline { 2 - 7 } \multicolumn{1}{c|}{} & Total\# & Length = 1 & Length = 2 & Length = 3 & Length = 4 & Length = 5 \\
\hline Technology Terms & 13,841 & 759 & 8,674 & 3,826 & 539 & 43 \\
\hline Valid Terms & 22,044 & 1,503 & 14,148 & 5,680 & 659 & 54 \\
\hline Invalid Terms & 61,758 & 15,887 & 33,474 & 11,016 & 1,210 & 171 \\
\hline \hline Total Annotated & 83,802 & 17,390 & 47,622 & 16,696 & 1,869 & 225 \\
\hline
\end{tabular}

Table 2: Summary statistics of the annotated candidate terms.

and the date (e.g. 1999, 2006, etc.) of publication.

The ACL RD-TEC is a spin-off of the ACL ARC corpus. It further enriches the ACL ARC metadata using automatic and manual annotations. The ACL RD-TEC employed the SectLabel module of Luong et al.'s (2010) ParsCit tool for the automatic identification of logical text sections in ACL ARC's raw text files. The resulting segmented text units are cleansed using a set of heuristics; for instance, broken words and text segments are joined, footnotes and captions are removed, and sections are organised into paragraphs. Text sections are further segmented into PoS-tagged sentences and each linguistically welldefined unit, e.g. types (i.e. PoS-tagged and lemmatized words), sentences, paragraphs and (sub)sections, is assigned to a unique identifier. These text units are stored and presented in inverted index files, in a tabseparated format. Hence, text units can be easily traced back to the contexts and, eventually, publications that they appeared in. Table 1 shows the statistics of text segments in the dataset.

The ACL RD-TEC consists of manual annotations that can be used for the evaluation of ATR and term classification tasks. In its current release, more than 80,000 lexical units ${ }^{7}$ are annotated as either valid or invalid terms. For a given lexical form $t$, if $t$ refers to a significant concept in the computational linguistics domain, it is annotated as valid. ${ }^{8}$ Examples of valid terms are 'natural language' and 'terminology'. In addition, valid terms are classified as those that can signal a technology concept. Technology terms indicate a method or a process that is employed to accomplish a task; examples of these terms are 'parsing' and 'information retrieval', and more delicate terms such as 'linear interpolation'.

Similar to the valid terms, terms that are annotated as technology terms do not exclusively belong to this class. For example, 'computational linguistics' is a lexical form that can be classified as a technology term, e.g., in '. . promising area of application of computational linguistics techniques...'. However, it can also signal other concepts such as a scientific discipline as well as a community, e.g., in '. . theoretical work in computational linguistics.. ' and '. . pursued by the computational linguistics community ...', respectively.

As reported in Zadeh and Handschuh (2014a), the observed agreement between 4 participants in the manual annotation of technology terms from a small set of randomly selected candidate terms is 0.828 ; the obtained Cohen's kappa coefficient for inter annotator agreement is 0.627 . Table 2 shows the current statistics of the annotated terms.

\subsection{Construction of Vector Space Models using Random Indexing}

We employ random indexing (RI) for the construction of the VSMs (Kanerva et al., 2000). For a corpus of a relatively small size, the context of terms can be represented and efficiently examined with the help of conventional vector space construction methods. The vector space is first constructed and then it may be followed by a dimensionality reduction technique. However, as the corpus grows and the number of elements that are employed for context definition increases, due to the high dimensionality of the vector space (orders of millions), these algorithms may suffer from low computational performance. $\mathrm{RI}$ is an approach that alleviates this problem by combining the construction of a vector space and the dimension reduction process. RI is based on normal random projection. It thus guarantees that

\footnotetext{
${ }^{7}$ A lexical unit is defined as a single token, part of a word, a word or a combination of these

${ }^{8}$ However, it is not guaranteed that all the occurrences of $t$ in the corpus are valid terms.
} 
the relative Euclidean distance between vectors, as well as their cosine similarity, in the original highdimensional vector space is preserved in the vector space that is constructed at a reduced dimensionality. The vector space construction is a two-step procedure: construction of (a) index vectors followed by the construction of (b) context vectors.

In the first step, each context element (i.e. a PoS-tagged word in a context window) is assigned exactly to one index vector. Index vectors are high-dimensional randomly generated vectors, in which most of the elements are set to 0 and only a few to 1 and $-1 .{ }^{9}$ Once an index vector is generated and assigned to a context element, this information is stored so that it can be retrieved and used for later analysis. In the second step, the construction of context vectors, each candidate term is assigned to a vector of which all elements are zero. This context vector has the same dimension as the index vectors. For each cooccurrence of a candidate term (represented by the context vector $\vec{v}_{t_{i}}$ ) and a context element (represented by the index vector $\vec{r}_{w_{j}}$ ) in the corpus, the context vector for the candidate term is accumulated by the index vector of the context element, i.e. $\vec{v}_{t_{i}}=\vec{v}_{t_{i}}+\vec{r}_{w_{j}}$. The corpus is scanned for all the cooccurrences of candidate terms and context elements and the context vectors are updated to reflect these co-occurrences. The result is a VSM that is constructed directly in the reduced dimensionality and represents candidate terms using the defined context.

For instance, in the example given in Figure 3, the term 'information extraction' is assigned to a context vector. If a context window of size 3 that extends in both directions of candidate terms and discards word order information is employed, then each unique word in the rectangles, e.g. has, current, technology, the, been, etc., is assigned to a randomly generated index vector. The context vector is then obtained by the accumulation of these index vectors. If the word order information is encoded, then the appearance of each word at a certain position in the context window must be assigned to a unique index vector. As a result, in the given example in Figure 3, the word 'technology' in the first sentence (position 1 after target term) and the third sentence (position 2 before the target term) is assigned to two different index vectors, each uniquely represents the word 'technology' at these positions.

In order to employ random indexing, two parameters must be decided: the dimensionality of the VSM and the number of non-zero elements. As described in Zadeh and Handschuh (2014c), using the provided proofs in Li et al. (2006), it can be verified that the dimensionality of RI-constructed VSMs is determined independently of the number of context elements $n$ (i.e. the original dimensionality of the vector space). It is, however, determined by the probability and the maximum expected amount of distortions in pairwise distances and the number of context vectors in the model (in logarithmic scale). The number of non-zero elements, on the other hand, is decided by the number of context elements and the sparseness of the VSM at the original high dimension $(\alpha)$ as $\mathrm{O}(\sqrt{\alpha n})$. Accordingly, in the reported experiment in this paper, we set the dimension of RI-constructed VSMs to 1800, which is large enough to make sure that the distances are preserved in the constructed VSMs. In our experimental setup using the contexts that are described in Section 2.4.1, the estimated non-reduced, original dimension of the vector space is between 700,000 and 7 million; ${ }^{10}$ hence, we set 8 elements of index vectors to \pm 1 .

\subsection{Term Scoring Method: $k$-Nearest Neighbours Regression}

We employ a standard $k$-nn regression method to assign scores to the extracted candidate terms. In this framework, context vectors that represent candidate terms are compared to those that represent a set of reference terms $R_{s} . R_{s}$ consists of both technology and non-technology terms that are manually tagged prior to the similarity measurement task. We employ the cosine similarity. For each candidate term $t$, terms in $R_{s}$ are sorted in descending order by their cosine similarity to $t$. We calculate the sum of the similarity of valid technology terms to $t$ in the top $k$ terms of this sorted list and consider it as a measurement of the technology-term class membership for $t$. As described later in Section 2.4.2, the

\footnotetext{
${ }^{9}$ This distribution of zero and \pm 1 elements in index vectors this leads to a Gaussian asymptotic distribution and consequently a Gaussian random projection matrix (see Zadeh and Handschuh, 2014c, for further explanation).

${ }^{10}$ In the defined contexts for our experiments, the original dimension of the vector space is determined by the number of types in the corpus, i.e. 700,000. This number increases when the word order information is also encoded. In the reported experiment, this number escalates to 7 million for a context window of size 5 that extends around the terms and encode word order information).
} 


\begin{tabular}{|c|c|c|c|c|c|c|}
\hline \#Term $_{\text {Total }}$ & \#Term $_{\text {Technology }}$ & Average Length & $f$ (Sentence) & $f$ (Paragraph) & $f$ (Section) & $f($ Document) \\
\hline 3,490 & 1,596 & 2.037 & $1,696,201$ & $1,264,616$ & 870,574 & 346,000 \\
\hline
\end{tabular}

Table 3: Summary statistics of the reference terms $R_{s}$. $f(x)$ denotes the accumulative frequency of occurrences of all the terms in $R_{s}$ in text segments of type $x$.

neighbourhood size $k$ is defined relative to the size of $R_{s}$.

As stated earlier, candidate terms are extracted from the ACL ARC corpus using a part-of-speechbased filtering technique. In this method, any sequence of tokens in the corpus that conforms to one of the predefined part-of-speech tag sequences is considered as a term candidate. By employing 31 different patterns of length 1 to 5 , we extract 1.3 million candidate terms. ${ }^{11}$ Using the $k$-nn regression described above, the technology-term class membership is calculated for all the candidate terms.

\subsubsection{Reference Term Formation}

Prior to the $k$-nn regression task, we extract all the candidate terms which ended or collocated with the words 'technology' and 'technique' (in their lemmatized form). Examples of the extracted terms are 'unsupervised text categorization', 'basic estimation', 'bi-directional bottom-up' and 'boolean keyword'. These terms are then manually annotated as technology and non-technology terms. For example, in the list of terms given above, only 'unsupervised text categorization' is annotated as a technology term. The process resulted in a set of reference terms $R_{s}$ consisting of 3490 terms of which 1596 are annotated as technology terms (i.e. positive examples). The accumulative frequency of the occurrences of the extracted reference terms in the corpus are given in Table 3.

\subsection{Evaluation Methodology}

In the reported evaluation framework, the procedure described in Section 2.2 is performed to construct several vector spaces of various context configurations, which are described in Section 2.4.1. The described procedure for term scoring in Section 2.3 is then employed to assign scores to the extracted candidate terms in all the constructed vector spaces. In each experiment, candidate terms are sorted in descending order by their assigned scores. The proportion of technology terms in the list of the top $n$ terms (we start with 250 terms) is reported for the comparison of the performance of the evaluated context configurations. We further investigate the role of the neighbourhood size selection $k$ as well as the number of reference terms $R_{s}$ in the performance of the scoring task.

\subsubsection{Evaluated Context Parameters}

In the reported evaluation, the terms' contexts are defined by the frequency of distinct PoS-tagged words that co-occurred with terms in a text window of limited size. We evaluate context windows that are configured with three parameters: direction, size and order.

The first parameter distinguishes context windows according to the direction in which they are expanded to collect the co-occurrence counts. The context window of a term is expanded (a) to the lefthand side of the term to count the co-occurrence counts of the term with its preceding words in each sentence of the corpus, (b) to the right-hand side to collect co-occurrences with the succeeding words or (c) around the term, i.e. in both left and right directions. The context windows are also configured by their size, i.e. the extent of terms' neighbourhood for counting the co-occurrences. As stated in Sahlgren (2008), an optimum size of a context window can only be found through experiments. However, he also suggests that if the goal is to capture a paradigmatic relation (such as the one proposed here), then narrow context windows outperform wide context windows. As a result, in our experiments we limit the size of context windows $w$ to $1 \leq w \leq 5$. For the context windows that expand around terms, we extend the context region symmetrically in both directions. As stated earlier, Figure 3 illustrates a context window of size 3 that extends around terms.

Some research suggests that the sequential order of words expresses information about the grammatical behaviour of words and, therefore, the inclusion of this information in a distributional model enhances

\footnotetext{
${ }^{11}$ The extracted candidate terms are included in the ACL RD-TEC.
} 
the performance. We investigate the impact of word order information on the performance of the suggested task. Capturing word order information requires distinguishing the location of words in context windows. To attain this goal in the random indexing technique, as stated earlier in Section 2.2, the appearance of the same word in different positions in a context window is recognized by assigning the word to several index vectors, each index vector denotes the appearance of the word in certain position. Alternatively, the order of words (i.e. the position of a word in a context window) can be captured by shuffling their index vectors via a permutation function (Sahlgren et al., 2008). ${ }^{12}$ In our implementation, a circular shift function serves as the permutation function. Accordingly, if $m$ is the number of tokens after/before a target term and a word in a context window, then the index vector of the word is shifted $m$ times circularly to the right/left before its accumulation to the target term's context vector.

\subsubsection{Evaluated Parameters of $k$-Nearest Neighbours Regression}

In addition to different configurations of context windows, we investigate the role of two other parameters in the performance of the proposed $k$-nn-based method: the neighbourhood size $k$ and the number of reference vectors $\left|R_{s}\right|$. The performance of $k$-nn is largely dependent on the value of $k$ : a small value for $k$ leads to over-fitting, while a large neighbourhood estimation may reduce the discriminatory power of the classifier. The optimum $k$ is subject to the number of reference vectors and the underlying probability distribution of target instances in the vector space. The underlying probability distribution is unknown and difficult to estimate. Therefore, the optimal value of $k$ is usually obtained by an experimental method (Yang, 1999). Yang also suggests that the performance of the $k$-nn algorithm is relatively stable for a large range of $k$ values. Accordingly, we perform an empirical assessment by inspecting the output of the proposed method with respect to various values of $k$ that are defined in relation to $\left|R_{s}\right|$. The main objective of our experiment, however, is to examine whether the best-performing context configuration can be distinguished irrespective of the value of $k$. Accordingly, we report the performance of the scoring procedure when $k=\left\lfloor p R_{s}\right\rfloor$, for $p \in\{0.001,0.005,0.01,0.1,0.2\}$.

Building reference vectors $R_{s}$ is laborious; it entails a manual annotation of terms. It requires a domain expert to provide a list of representative technology terms (positive examples) and nontechnology terms (negative examples) from the corpus that is being analyzed. ${ }^{13}$ As a result, a $R_{s}$ of small size is often more desirable than a large one. In $k$-nn, a small $R_{s}$ is also desirable from the computational complexity point of view. However, using a large set of reference vectors often yields higher performance. As a result, the choice of the number of reference vectors $\left|R_{s}\right|$ is a trade-off between efficiency and performance. We thus compare the performance of the method for values of $\left|R_{s}\right| \in\{100,200,300,600,1100,1600,3200,3490\}$. In each experiment, we made sure that the created $R_{s}$ has a balanced number of positive and negative examples; however, the terms are chosen randomly.

\section{Observed Evaluation Results}

Table 4 reports the observed results in the first set of experiments. We start to score all the candidate terms using the complete set of reference vectors, i.e. $\left|R_{s}\right|=3490$. We perform the experiments for all the possible configurations of context windows, as described in Section 2.4. Each of these experiments are repeated for $k=\left\lfloor p\left|R_{s}\right|\right\rfloor$, for $p \in\{0.001,0.005,0.01,0.1,0.2\}$. In these experiments, therefore, the assessed values of $k$ are $\{3,17,34,349,698\}$. Table $4 \mathrm{a}$ and $4 \mathrm{~b}$ shows the observed results in the constructed VSMs when the word order information is excluded and included, respectively. In both tables, columns show the observed proportion of technology terms in the top 250 terms in the list of candidate terms that are weighted using the proposed method; thus, the closer a number is to 1 , the higher the performance. We suggest Frantzi et al.'s (1998) $c$-value score-a general ATR algorithmfor the baseline measure. The $c$-value score of a term is measured by its frequency in the corpus that is normalized by its length and the frequency of its occurrences in other longer terms as a nested term. In our experiment, the proportion of technology terms in the top 250 terms in the list of candidate terms that are weighted by the $c$-value score is 0.252 .

\footnotetext{
${ }^{12}$ Assuming that shuffling of index vectors is equivalent to generating a new one.

${ }^{13}$ Depending on the type of classification-regression technique, the negative examples may not be required.
} 


\begin{tabular}{|c|c|c|c|c|c|c|}
\hline \multicolumn{2}{|c|}{ Context } & \multicolumn{3}{|c|}{ Value of $\mathbf{p}$ in $\mathbf{k}=\left\lfloor p \mid R_{s}\right.$} & \multicolumn{2}{|c|}{$=\lfloor p \cdot 3400\rfloor$} \\
\hline Type & Size & 0.001 & 0.005 & 0.01 & 0.10 & 0.20 \\
\hline \multirow{5}{*}{ בَّత } & 1 & 0.264 & 0.592 & 0.596 & 0.580 & 0.536 \\
\hline & 2 & 0.132 & 0.688 & 0.664 & 0.644 & 0.556 \\
\hline & 3 & 0.376 & 0.688 & 0.696 & 0.592 & 0.512 \\
\hline & 4 & 0.524 & 0.692 & 0.656 & 0.616 & 0.536 \\
\hline & 5 & 0.636 & 0.660 & 0.660 & 0.580 & 0.504 \\
\hline \multirow{5}{*}{$\underset{00}{\overrightarrow{0}}$} & 1 & 0.124 & 0.440 & 0.512 & 0.224 & 0.284 \\
\hline & 2 & 0.628 & 0.688 & 0.288 & 0.380 & 0.360 \\
\hline & 3 & 0.684 & 0.496 & 0.440 & 0.380 & 0.380 \\
\hline & 4 & 0.596 & 0.664 & 0.340 & 0.456 & 0.412 \\
\hline & 5 & 0.708 & 0.632 & 0.672 & 0.452 & 0.436 \\
\hline \multirow{5}{*}{ 吾 } & $\mathbf{1}$ & 0.440 & 0.748 & 0.744 & 0.608 & 0.616 \\
\hline & 2 & 0.716 & 0.836 & 0.848 & 0.764 & 0.700 \\
\hline & 3 & 0.760 & 0.840 & 0.816 & 0.696 & 0.664 \\
\hline & 4 & 0.160 & 0.800 & 0.788 & 0.672 & 0.660 \\
\hline & 5 & 0.144 & 0.748 & 0.740 & 0.660 & 0.660 \\
\hline
\end{tabular}

(a) No Word Order Information

\begin{tabular}{|c|c|c|c|c|}
\hline \multicolumn{5}{|c|}{ Value of $\mathbf{p}$ in $\mathbf{k}=\left\lfloor p\left|R_{s}\right|\right\rfloor=\lfloor p \cdot 3400\rfloor$} \\
\hline $\mathbf{0 . 0 0 1}$ & $\mathbf{0 . 0 0 5}$ & $\mathbf{0 . 0 1}$ & $\mathbf{0 . 1 0}$ & $\mathbf{0 . 2 0}$ \\
\hline 0.264 & 0.592 & 0.596 & 0.580 & 0.536 \\
0.128 & 0.660 & 0.644 & 0.588 & 0.524 \\
0.344 & 0.676 & 0.652 & 0.544 & 0.448 \\
0.400 & 0.648 & 0.664 & 0.524 & 0.436 \\
0.448 & 0.632 & 0.640 & 0.488 & 0.408 \\
\hline \hline 0.124 & 0.440 & 0.512 & 0.224 & 0.284 \\
0.696 & 0.736 & 0.788 & 0.316 & 0.368 \\
0.720 & 0.388 & 0.688 & 0.304 & 0.328 \\
0.580 & 0.376 & 0.600 & 0.324 & 0.328 \\
0.768 & 0.384 & 0.552 & 0.328 & 0.328 \\
\hline \hline 0.376 & 0.768 & 0.804 & 0.704 & 0.624 \\
\hline 0.796 & 0.836 & 0.824 & 0.644 & 0.608 \\
0.760 & 0.828 & 0.808 & 0.636 & 0.572 \\
0.784 & 0.808 & 0.776 & 0.612 & 0.540 \\
0.784 & 0.808 & 0.756 & 0.580 & 0.516 \\
\hline
\end{tabular}

(b) Encoded Word Order Information

Table 4: The observed results from the performed evaluations. The number columns show the proportion of technology terms in the top 250 terms for various values of $k$.

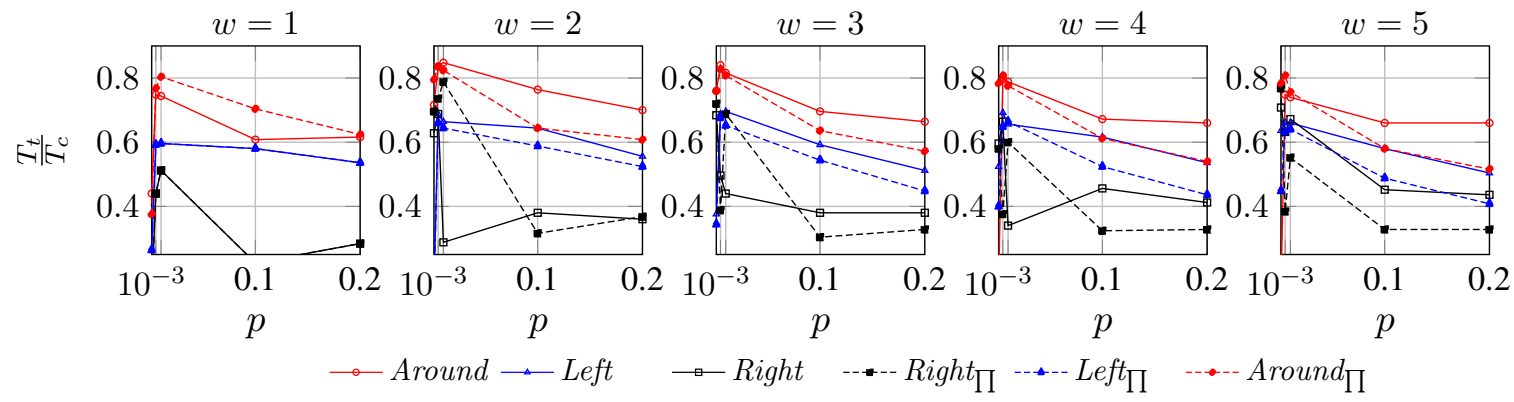

Figure 5: The performance of various context configurations over various neighbourhood sizes of $k=$ $\left\lfloor p\left|R_{s}\right|\right\rfloor$. $\left|\frac{T_{t}}{T_{c}}\right|$ denotes the proportion of technology terms in the top 250 terms that are sorted by their assigned scores. $\prod$ denotes context types that encode word order information. The minimum value of $\left|\frac{T_{t}}{T_{c}}\right|$ axis is set to 0.252 , i.e. our baseline. The baseline is the proportion of technology terms in the top 250 terms in the list of candidate terms that are weighted using the $c$-value technique.

As shown in Figure 5, weighting terms in the VSMs that are built using context windows that extend in both directions of candidate terms (i.e. around candidate terms) outperforms VSMs constructed by other types of context windows. In addition, as can also be verified in Table 4, the results from narrow context windows $(1 \leq w \leq 3)$ are more desirable than those from wide context windows ( $w \geq 3)$. However, in contrast to our previous experiments, in which we employed an unweighted $k$-nn voting classification framework (Zadeh and Handschuh, 2014b), encoding word order information in the constructed VSMs does not necessarily improve the results. It is important to note that for different values of $k$, although the overall performance of the TTR method changes, the relative performance of the employed context windows with respect to each other is, nearly, constant. Therefore, we conclude that the best-performing context type, thus VSM, can be decided independently of the value of $k$ : a result similar to that reported in Zadeh and Handschuh (2014b) for an unweighted $k$-nn voting classification.

\subsection{Inspecting the Effect of Reference Vector Size}

We are interested in studying the effect of reference vector size, i.e. $\left|R_{s}\right|$, on the overall performance of the technology term recognition task. In this set of experiments, we limit our evaluation to the bestperforming context window in the previous evaluation task, i.e. the context window that extends around candidate terms. We repeat the scoring process for $\left|R_{s}\right| \in\{100,200,300,600,1600,3490\}$. Similar to the previous set of experiments, we define and express the neighbourhood size $(k)$ with respect to the 


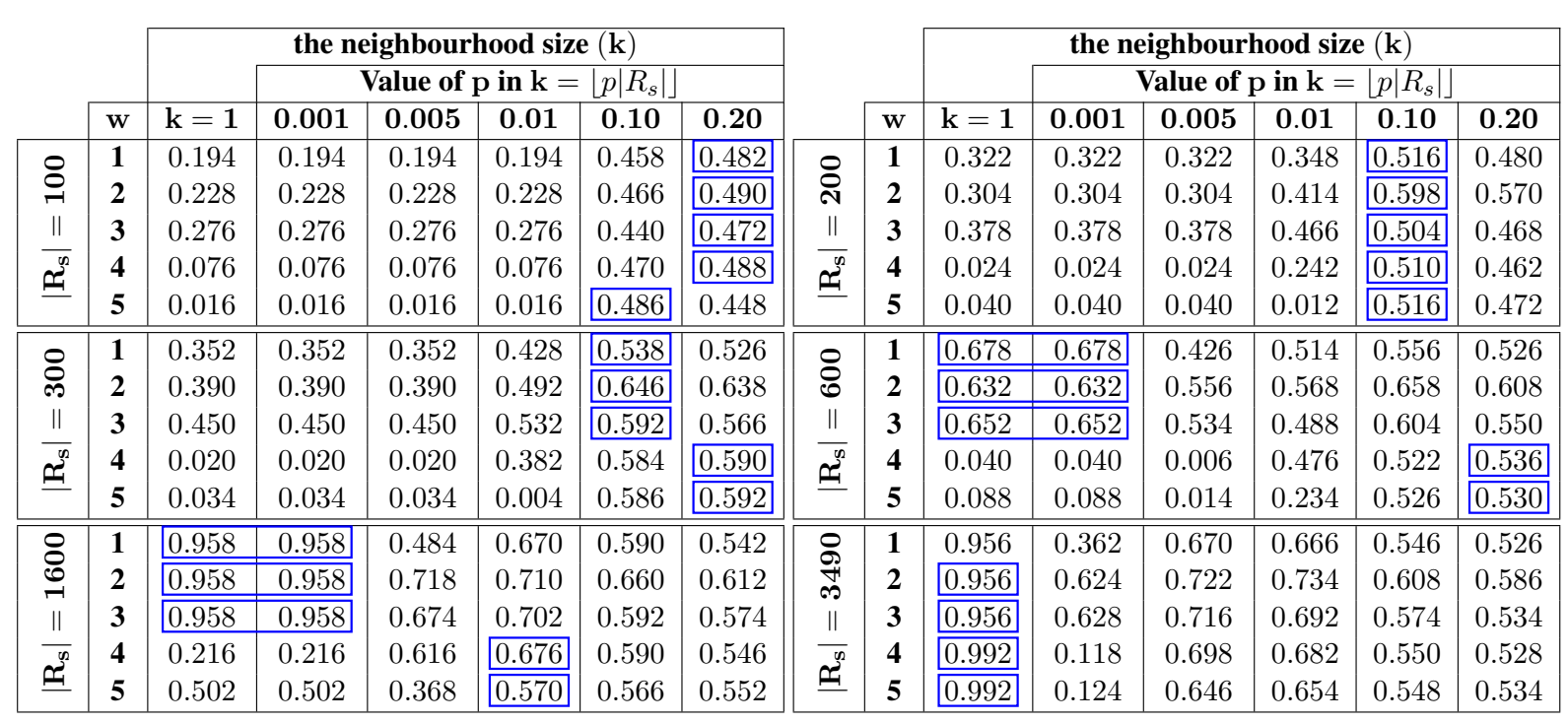

Table 5: The observed results, i.e. the proportion of technology terms in the list of top 500 candidate terms, for various sizes of the reference vectors set $\left(\left|R_{s}\right|\right)$ and the neighbourhood size $(k)$ for the context window that extends around the terms; $w$ denotes the size of context window.

size of the reference vectors $\left(\left|R_{s}\right|\right)$, i.e. $k=\left\lfloor p\left|R_{s}\right|\right\rfloor$, for $p \in\{0.001,0.005,0.01,0.1,0.2\}$. In addition, we report the results for to the nearest-neighbour algorithm, i.e. when $k=1$. For $\left|R_{s}\right|<1000$ and $p=0.001$ that results to $k=\left\lfloor 0.001\left|R_{s}\right|\right\rfloor=0$, we set $k=1$. In these cases, thus, the reported results for $k=\left\lfloor p\left|R_{s}\right|\right\rfloor$ is equivalent to the results reported for $k=1$.

Table 5 reports the observed results: the proportion of technology terms in the top 500 terms in the list of candidate terms. First, these results suggest that the optimum value of $p$ in $k=\left\lfloor p\left|R_{s}\right|\right\rfloor$, and thus $k$, depends on $\left|R_{s}\right|$. If $\left|R_{s}\right|$ is small, a larger neighborhood performs better than a smaller neighborhood. Inversely, if $\left|R_{s}\right|$ is large enough, a small neighbourhood shows higher performance than a large neighborhood. Second, a small neighbourhood is sensitive to the size of context window $w$ (and perhaps the presence of noise), specifically when $\left|R_{s}\right|$ is small. As an example, for $\left|R_{s}\right|=600$ and $k=1$, if $w \geq 4$, then the performance drops sharply. Therefore, the performance of large neighbourhoods can be more stable than the performance of small neighbourhoods. Lastly, when $\left|R_{s}\right|$ reaches 1600 (a certain threshold), there is no significant increase in the performance of the algorithm. In this case, the nearest-neighbour algorithm outperforms the $k$-nn method. We suggest that the obtained results from the nearest-neighbour method can be used as a heuristic-based strategy for the selection of the number of vectors in the $\mid R_{\mid}$. Accordingly, one can stop adding new vectors to $R_{s}$ when the obtained results from the nearest-neighbour method are above a certain threshold.

\section{Conclusion}

In this paper, we proposed a corpus-based, distributional method for the recognition of technology terms from a corpus of scientific publications. The method is established as a $k$-nn regression task in a Euclidean vector space, in which vectors are compared by their cosine similarity. This vector space represents the co-occurrence frequencies of candidate terms and words in a context window. We examined a number of factors that play roles in the performance of the proposed method.

In order to find the most discriminative models, we studied several configurations of the context window: its size, the direction in which it is extended, and the incorporation of the word order information. According to these experiments, context windows that collect co-occurrence frequencies in both sides of terms, in narrow context, i.e. the size of 2 or 3 words, outperform other context types. We observed that narrow context windows, irrespective of other variables in the model (i.e. the $k$ and the number of reference vectors), consistently show a performance higher than other context configurations. Therefore, we suggest that the best-performing context type can be decided independently of the value of $k$ and 
the reference vector size. We also reported an initial experiment that assessed the effect of the reference vector size on the performance of the system.

We performed our evaluations on the ACL ARC corpus. Apart from the proposed methodology and reported experiments, another outcome of the performed experiment is a relatively large set of annotated technology terms, i.e. the ACL RD-TEC. The annotated terms in the ACL RD-TEC can be easily mapped into the ACL ARC documents, thus, into a chronological order and in a citation network. As a result, the annotations resulting from the experiments reported in this paper can be used in tasks other than technology term recognition, e.g. citation analysis and technology forecasting.

The reported experiment can be extended in several ways. In this paper, we focused on the extraction of technology terms at the corpus level. It would be helpful to investigate the best-performing context configurations when the co-occurrences are collected from communicative contexts of other sizes than corpus, e.g. at a document level, similar to automatic keyphrase extraction tasks. It is also interesting to compare the performance of the $k$-nn instance-based algorithm with other learning techniques such as support vector machines. Last but not least, we are interested in re-evaluating the proposed method using metrics other than the top $n$ terms look-up in the sorted weighted list of terms. A comparison of the evaluation metrics could be an attractive research avenue.

\section{Acknowledgements}

We thank the anonymous reviewers. This publication has emanated from research conducted with the financial support of Science Foundation Ireland under Grant Number SFI/12/RC/2289.

\section{References}

Steven Bird, Robert Dale, Bonnie Dorr, Bryan Gibson, Mark Joseph, Min-Yen Kan, Dongwon Lee, Brett Powley, Dragomir Radev, and Yee Fan Tan. 2008. The ACL anthology reference corpus: a reference dataset for bibliographic research in computational linguistics. In LREC'08. Marrakech, Morocco.

Stefan Evert. 2004. The Statistics of Word Cooccurrences Word Pairs and Collocations. Ph.D. thesis, Institut für maschinelle Sprachverarbeitung, Universität Stuttgart.

KaterinaT. Frantzi, Sophia Ananiadou, and Junichi Tsujii. 1998. The $c$-value/nc-value method of automatic recognition for multi-word terms. In Research and Advanced Technology for Digital Libraries, volume 1513 of LNCS, pages 585-604.

Zellig S. Harris. 1954. Distributional structure. Word, The Journal of the International Linguistic Association, 10:146-162.

Kyo Kageura and Bin Umino. 1996. Methods of automatic term recognition: A review. Terminology, 3.2 (1996):259-289.

Pentti Kanerva, Jan Kristoferson, and Anders Holst. 2000. Random indexing of text samples for latent semantic analysis. In Proceedings of the 22nd Annual Conference of the Cognitive Science Society, pages 103-6. Erlbaum.

Alessandro Lenci. 2008. Distributional semantics in linguistic and cognitive research. From context to meaning: Distributional models of the lexicon in linguistics and cognitive science, special issue of the Italian Journal of Linguistics, 20/1:1-31.

Ping Li, Trevor J. Hastie, and Kenneth W. Church. 2006. Very sparse random projections. In Proceedings of the 12th ACM SIGKDD International Conference on Knowledge Discovery and Data Mining, KDD '06, pages 287-296. ACM, NY, USA.

Minh-Thang Luong, Thuy Dung Nguyen, and Min-Yen Kan. 2010. Logical structure recovery in scholarly articles with rich document features. IJDLS, 1(4):1-23.

Nils C. Newman, Alan L. Porter, David Newman, Cherie Courseault Trumbach, and Stephanie D. Bolan. 2014. Comparing methods to extract technical content for technological intelligence. JET-M, 32(0):97 - 109.

Behrang QasemiZadeh. 2010. Towards technology structure mining from text by linguistics analysis. DERI technical report, National University of Ireland, Galway.

Magnus Sahlgren. 2008. The distributional hypothesis. Italian Journal of Linguistics, 20:33-54.

Magnus Sahlgren, Anders Holst, and Pentti Kanerva. 2008. Permutations as a means to encode order in word space. In V. Sloutsky, B. Love, and K. Mcrae, editors, Proceedings of the 30th Annual Conference of the Cognitive Science Society, pages 1300-1305. Cognitive Science Society, Austin, TX.

Peter D. Turney and Patrick Pantel. 2010. From frequency to meaning: vector space models of semantics. J. Artif. Int. Res., 37(1):141-188.

Yiming Yang. 1999. An evaluation of statistical approaches to text categorization. Inf. Retr., 1(1-2):69-90.

Behrang Q. Zadeh and Siegfried Handschuh. 2014a. The ACL RD-TEC: a dataset for benchmarking terminology extraction and classification in computational linguistics. In Coling 2014 CompuTerm 2014. Dublin, Ireland.

Behrang Q. Zadeh and Siegfried Handschuh. 2014b. Evaluation of technology term recognition with random indexing. In LREC'14. European Language Resources Association (ELRA), Reykjavik, Iceland.

Behrang Q. Zadeh and Siegfried Handschuh. 2014c. Random manhattan indexing. Database and Expert Systems Applications (DEXA), 25th International Workshop on. IEEE, Munich, Germany. 